\title{
CYCLE ÉVOLUTIF D’UN CESTODE PROTEOCEPHALIDAE PARASITE DU CORÉGONE DU LAC LÉMAN (COREGONUS LAVARETUS L.)
}

\author{
Étude du parasite dans le milieu naturel \\ et dans des conditions expérimentales
}

\author{
H. MORANDI*, D. PONTON**
}

RÉSUMÉ. Le cycle saisonnier de développement du Cestode Proteocephalidae du genre Proteocephalus, parasite du corégone du lac Léman comprend une phase de maturité sexuelle des individus allant de juin à septembre, suivie par une disparition des parasites adultes en hiver.

Les tentatives de reconstitution du cycle parasitaire au laboratoire ont montré l'importance de la densité des hôtes intermédiaires (Cyclops vicinus Ulianine, 1875) pour la réussite des infestations ainsi que de paramètres comme la maturité des œufs et la température de l'eau.

L'infestation artificielle de corégones $0^{+}$prouve que la réalisation du cycle complet de ce parasite est possible au laboratoire. Les différentes phases de ce cycle sont décrites. Le protéocéphale des corégones du lac Léman semble pouvoir être un bon marqueur biologique du flux trophique entre le zooplancton et les poissons de cette espèce en hiver et au printemps.

Mots-clés : Proteocephalus sp. Cycle biologique. Coregonus lavaretus L. Lac Léman.

\section{Cycle of a proteocephalan Cestode of whitefish (Coregonus lavaretus L.) from Lake} Léman. Parasite study in the natural environment and under experimental conditions.

SUMMARY. The seasonal cycle of the proteocephalan Cestode, genus Proteocephalus, found in whitefish (Coregonidae) from Lake Léman, presents a period of sexual maturity between June and September. Adult worms disappear in winter.

Studies of the cycle in laboratory conditions have shown the importance of first host densities (Cyclops vicinus Ulianine, 1875) for the success of infestations as well as of factors such as eggs maturity and water temperature.

Artificial infestations of $0^{+}$whitefish have proved that successful completion of the whole cycle is possible in a laboratory. The different phases of this cycle are described hereafter.

The proteocephalan parasite of whitefish from Lake Léman seems to be a good biological tag for the assessment of food requirements of these fish in winter and spring.

Key-words: Proteocephalus sp. Life cycle. Whitefish. Coregonus lavaretus L. Lake Léman.

* Centre National de Formation des Techniciens des Services Vétérinaires. Z. I. de CorbasMontmartin, F 69960 Corbas.

** Seenforschungslaboratorium der EAWAG/ETH, CH-6047 Kastanienbaum.

Accepté le 13 décembre 1988. 


\section{Introduction}

En 1964, Doby et Jarecka ont décrit un Cestode Proteocephalidae du genre Proteocephalus parasite du corégone du lac Léman. En 1965, Jarecka et Doby rapprochent ce Protéocéphale des espèces Proteocephalus neglectus (La Rue, 1911) et Proteocephalus pollanicola et tentent une reconstitution du cycle évolutif de ce parasite. Ils ont montré le rôle des Copépodes Cyclopidae comme premiers hôtes intermédiaires. Chaubeau-Duffour et Morandi (1984) ont observé une forme larvaire d'un Cestode Proteocephalidae chez des corégones pêchés dans ce même lac en 1983.

Les caractéristiques de ce parasite et du groupe auquel il appartient, en particulier sa grande spécificité parasitaire, en font un matériel intéressant pour une étude de son rôle éventuel de marqueur biologique entre le compartiment zooplanctonique et les poissons planctonophages que sont les corégones (Iyeshko et al., 1981).

Un tel objectif demande un approfondissement de la connaissance du matériel parasitaire, de ses cycles biologique et saisonnier. Cette approche s'est développée dans trois domaines complémentaires :

- Tout d'abord une étude du cycle saisonnier de ce Cestode Proteocephalidae dans les tubes digestifs des corégones du lac Léman effectuée en 1986 et 1987.

- Ensuite, une étude quantitative de la première partie du cycle et plus particulièrement de l'influence de la densité des Copépodes Cyclopides sur la réussite de leur infestation.

- Enfin, une tentative de reconstitution de la totalité du cycle parasitaire depuis les adultes ovigères prélevés chez les corégones jusqu'à l'infestation d'individus $0^{+}$de cette même espèce en passant par une infestation artificielle des Copépodes.

\section{Matériel et méthode}

Pour chaque expérimentation, et d'après les définitions de Margolis et al. (1982), les grandeurs observées sont :

$$
\begin{aligned}
& \text { La prévalence }=\frac{\text { Nombre d'individus parasités }}{\text { Nombre d'individus examinés }} \times 100 \\
& \text { L'intensité }=\frac{\text { Nombre de parasites observés }}{\text { Nombre d'individus parasités }}
\end{aligned}
$$




\section{1 - Cycle saisonnier}

Le développement du parasite dans le tube digestif de corégones adultes a été déterminé à partir de poissons provenant de la pêche professionnelle à différentes périodes de l'année en 1986 et 1987. Ces observations ont été complétées par quelques données obtenues en 1984 et 1985 de la même manière.

Pour chaque pêche, les prévalences des différents stades de développement (juvénile, protéocéphale non segmenté, protéocéphale segmenté non ovigère et protéocéphale ovigère) ont été déterminés.

\section{2 - Première partie du Gycle : infestation de Copépodes}

Cette expérience a 'eu lieu en juillet-août 1986, c'est-à-dire durant la période de maturité sexuelle du matériel parasitaire.

Les Cestodes ovigères ont été récoltés dans des tubes digestifs de corégones issus de la pêche professionnelle le 29 juillet 1986. Après dissection de la zone intestinale, les parasites ont été immergés dans de l'eau du lac filtrée (température de l'eau : $23^{\circ} \mathrm{C}$ ), où ils libèrent spontanément leurs œufs par les pores génitaux. Après un séjour de 15 à 30 minutes dans l'eau, un même nombre d'embryons (120000 embryons \pm 2000$)$ a été mis en contact avec des Cyclopides répartis dans quatre bacs présentant deux densités d'individus différentes (tableau I). Deux bacs supplémentaires constituaient les bacs témoins. Après 17 jours où les conditions thermiques sont restées stables (température de l'eau : $23^{\circ} \mathrm{C} \pm 1$ ) la prévalence et l'intensité des larves de Protéocéphales ont été déterminées chez tous les Cyclopides survivants.

Tableau I. - Densités de Cyclopides (copépodites et adultes) dans les bacs expérimentaux. $\mathrm{D}^{-}$: bacs de faible densité $; \mathrm{D}^{+}$: bacs de forte densité $; \mathrm{T}$ : bacs témoins ; m : moyenne ; e.-t. : écart-type.

\begin{tabular}{cccccc}
\hline \multirow{2}{*}{ Bacs } & \multicolumn{2}{c}{ Copépodites } & & \multicolumn{2}{c}{ Adultes } \\
\cline { 2 - 5 } \cline { 5 - 6 } & $\mathrm{m}$ & e.-t. & & $\mathrm{m}$ & e.-t. \\
\hline $\mathrm{D}^{-}$ & 2650 & 610 & & 550 & 150 \\
\hline $\mathrm{D}^{+}$ & 13250 & 3060 & 2750 & 770 \\
\hline $\mathrm{T}$ & 13250 & 3060 & 2750 & 770 \\
\hline
\end{tabular}

\section{3 - Reconstitution de la totalité du Cycle parasitaire}

Des Cyclopides ont été infestés de la même manière que précédemment le 24 juillet 1987 (température de l'eau : 18,50 C). Après 3, 6, 10 et 14 jours, des prélèvements de Cyclopides ont été effectués pour contrôler l'évolution de l'infes- 
tation. Au bout de 15 jours, les individus survivants ont été donnés comme nourriture à deux lots de 20 corégones $0^{+}$placés en isolement depuis 24 heures dans des aquariums alimentés en eau de source (température de l'eau : $18^{\circ} \mathrm{C}$ ). Trente autres individus, placés dans les mêmes conditions, constituaient le lot témoin. Les poissons ont été nourris à l'aide d'un aliment artificiel jusqu'au 20 décembre 1987, date à laquelle leur autopsie a été pratiquée pour déterminer la prévalence et l'intensité de l'infestation par les protéocéphales.

\section{Résultats}

\section{1 - Cycle saisonnier}

Toutes les données provenant de différentes années ont été regroupées (tableau II). La période durant laquelle les parasites sont ovigères va de juin à novembre. Les parasites adultes disparaissent ensuite pendant l'hiver et seules persistent des formes juvéniles dont la structure est comparable à celle de la larve parasite des Cyclopides. Au printemps, on observe une maturation progressive du parasite; il s'allonge tout en conservant sa structure de larve. Par la suite, il développe un appareil génital d'abord mâle, puis femelle au fur et à mesure de sa croissance.

Tableau II. - Prévalence des différents stades de développement du parasite dans la zone intestinale chez le corégone du lac Léman. $\mathrm{N}$ : nombre de poissons analysés.

\begin{tabular}{|c|c|c|c|c|c|c|}
\hline \multirow[t]{2}{*}{ Date } & \multirow[t]{2}{*}{$\mathrm{N}$} & \multirow{2}{*}{$\begin{array}{c}\text { Age } \\
\text { des } \\
\text { poissons }\end{array}$} & \multicolumn{4}{|c|}{ Prévalences } \\
\hline & & & $\begin{array}{l}\text { Juvé- } \\
\text { niles }\end{array}$ & $\begin{array}{l}\text { Protéo- } \\
\text { céphale } \\
\text { non } \\
\text { segmenté }\end{array}$ & $\begin{array}{c}\text { Protéo- } \\
\text { céphale } \\
\text { non } \\
\text { ovigère }\end{array}$ & $\begin{array}{l}\text { Protéo- } \\
\text { céphale } \\
\text { ovigère }\end{array}$ \\
\hline $12-1985$ & 24 & $1^{+}, 2^{+}$ & 16 & 0 & 0 & 4 \\
\hline 03-1986 & 8 & $1^{+}, 2^{+}$ & 0 & 50 & 0 & 0 \\
\hline 06-1985 & 10 & $1^{+}, 2^{+}$ & 0 & 0 & 80 & 50 \\
\hline 07-1987 & 6 & $2^{+}, 3^{+}$ & 0 & 0 & 0 & 40 \\
\hline 08-1987 & 30 & $2^{+}, 3^{+}$ & 0 & 0 & 0 & 40 \\
\hline $09-1986$ & 6 & $1^{+}, 2^{+}, 3^{+}$ & 0 & 0 & 0 & 100 \\
\hline 11-1986 & 18 & $1^{+}, 2^{+}, 3^{+}$ & 0 & 0 & 0 & 100 \\
\hline
\end{tabular}

\section{2 - Infestation artificielle de Cyclopides}

Tous les Copépodes infestés (tableau III) ont été identifiés comme étant Cyclops vicinus Ulianine, 1875. La prévalence naturelle correspondant à celle observée pour les lots témoins est de 0,51. Les comparaisons des prévalences entre les bacs à faible densité $\left(\mathrm{D}^{-}\right)$ou à forte densité de Cyclopides $\left(\mathrm{D}^{+}\right)$et les bacs témoins (T) montrent que la densité de Copépodes doit être faible pour que la prévalence augmente de façon significative (tableau IV). 
Tableau III. - Prévalence et intensité de l'infestation des Cyclopides. Légende identique à celle du tableau I.

\begin{tabular}{ccccc}
\hline Bac & Prévalence & Intensité & \multicolumn{2}{c}{ Longueur des larves $(\mu \mathrm{m})$} \\
\cline { 4 - 5 } & & & $\mathrm{m}$ & e.-t. \\
\hline D & 7,1 & 2,62 & 366 & 94 \\
$\mathrm{D}^{-2}$ & 8,4 & 2,77 & 315 & 58 \\
$\mathrm{D}^{+} 1$ & 1,7 & 1,33 & 425 & 64 \\
$\mathrm{D}^{+2}$ & 2,3 & 1,00 & 420 & 156 \\
$\mathrm{~T} 1$ & 0,6 & 1,00 & - & - \\
$\mathrm{T} 2$ & 0,4 & 1,00 & - & - \\
\hline
\end{tabular}

TABleau IV. - Comparaison des prévalences observées pour les différents bacs expérimentaux. $p$ : risque de premier ordre associé à Ho : "Les prévalences sont identiques $"$.

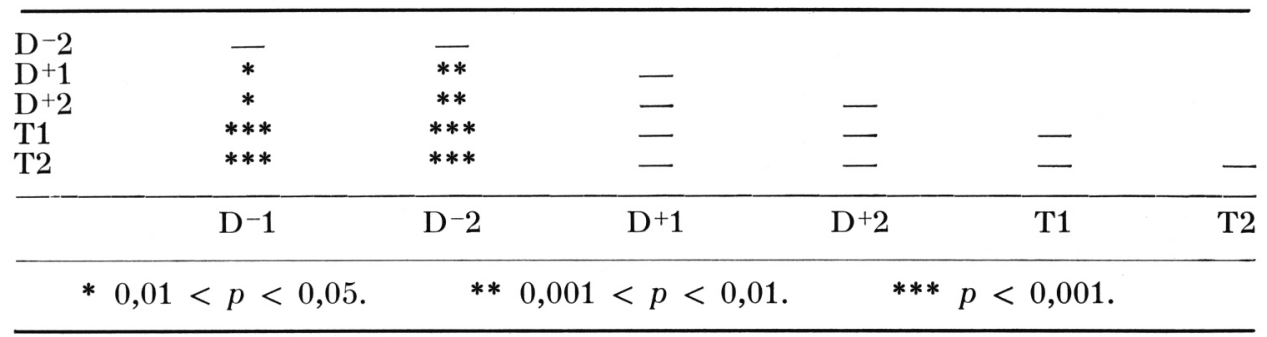

\section{3 - Reconstitution du GyCle Complet ET DESCRiption DES DiffÉRENTS STADES DE DÉVELOPPEMENT}

\section{1 - LES GUFS}

Après la ponte, l'observation des œufs sous microscope permet de déterminer deux cas :

- des œufs intacts, turgescents, avec une onchosphère en place dans son enveloppe et dans les deux enveloppes interne et externe de l'œuf (fig. 1) ;

- des œufs dont l'onchosphère a percé son enveloppe et l'enveloppe interne de l'œuf pour être libre dans l'enveloppe externe toujours turgescente (fig. 2).

Cette " éclosion " semble être un phénomène physiologique nécessaire à la réussite de l'infestation plutôt qu'à l'effet de la pression de la lamelle sur les œufs. En effet, l'enveloppe externe, turgescente, devrait être la première à se rompre en cas d'augmentation brutale de la pression. De plus, si on augmente le volume d'eau entre lame et lamelle, ces «éclosions " se poursuivent à une cadence régulière. Les différentes caractéristiques morphologiques de ce stade sont présentées au tableau $V$. 


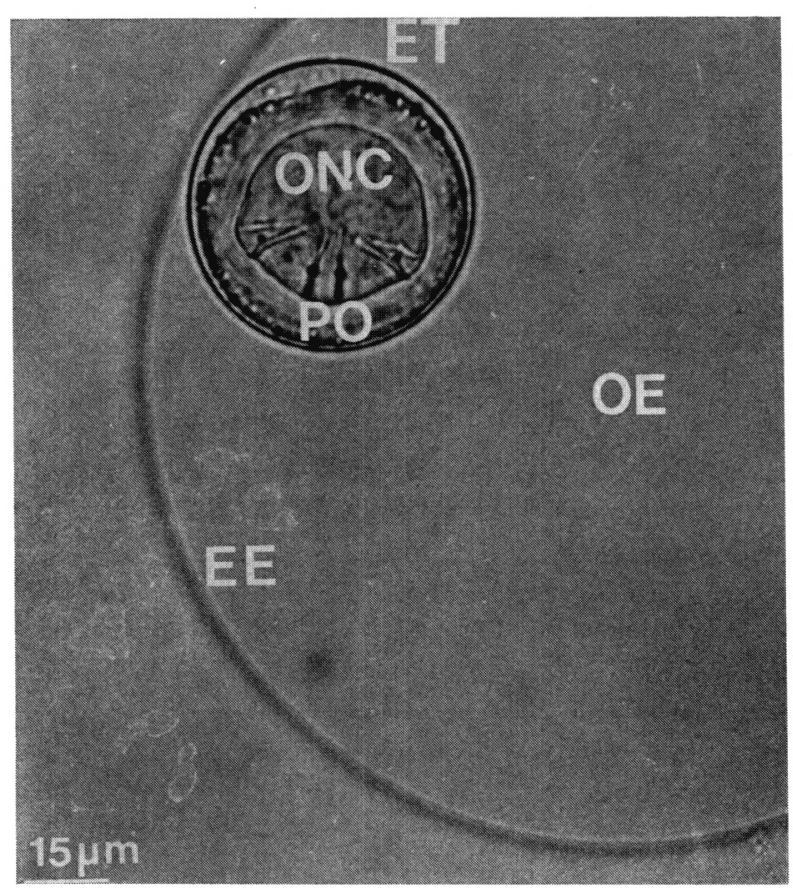

Fig. 1. - Euf intact avec son onchosphère en place dans son enveloppe et dans les deux enveloppes interne et externe de l'œuf. EE : enveloppe externe ; EI : enveloppe interne ; OE : œuf ; $\mathrm{ONC}$ : onchosphère ; PO : paroi de l'onchosphère.

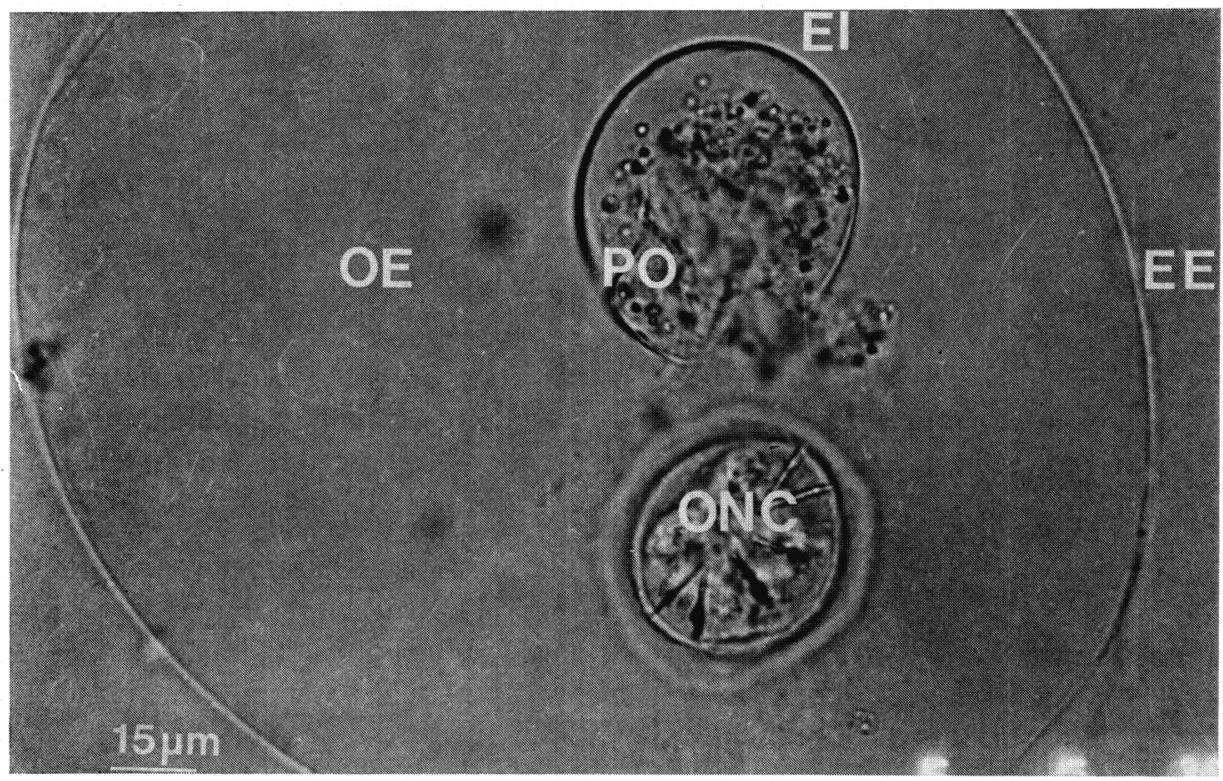

FIG. 2. - Onchosphère ayant percé son enveloppe et l'enveloppe interne de l'œuf. Légende identique à celle de la figure 1 . 
Tableau V. - Caractéristiques morphologiques des œufs.

Diamètre de l'œuf

Diamètre de la membrane interne

Diamètre de l'onchosphère

Longueur des crochets latéraux

Longueur des crochets médians

$$
\begin{array}{r}
140-160 \mu \mathrm{m} \\
40 \mu \mathrm{m} \\
25-28 \mu \mathrm{m} \\
8-9 \mu \mathrm{m} \\
10-12 \mu \mathrm{m}
\end{array}
$$

\section{2 - Les larves parasites de Cyclops vicinus}

Après trois jours, les Cyclops infestés (nombre d'observations : 10 ; prévalence : 100) montrent des embryons possédants encore leurs six crochets et placés dans la cavité générale de l'hôte, près du tube digestif. Leur diamètre n'est pas supérierur à celui de l'onchosphère dans l'œuf c'est-à-dire 24 à $27 \mu \mathrm{m}$.

Après six jours, les larves ont une longueur d'une centaine de microns et portent un cercomère (nombre d'observations : 10 ; prévalence : 100 ; intensité : 5). Les ventouses latérales ne sont marquées que par une légère dépression et la ventouse apicale par un massif cellulaire. Quelques rares granulations et deux crochets résiduels sont visibles.

Après 10 jours (nombre d'observations : 10, prévalence : 100 ; intensité : 5 ), les ventouses sont bien visibles, y compris la ventouse apicale (fig. 3). Des

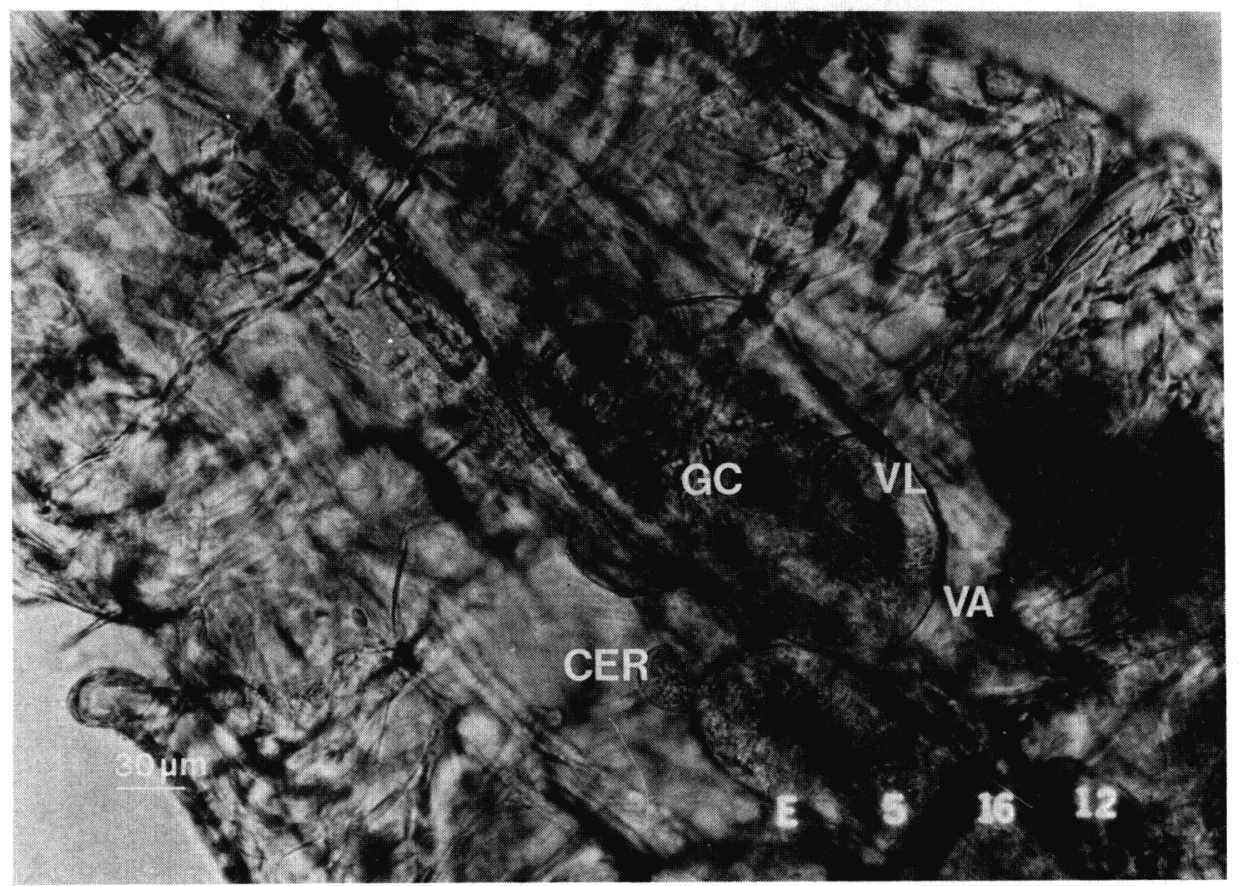

Fig. 3. - Larves de protéocéphale dans la cavité générale d’un Cyclops vicinus 10 jours après l'infestation. CER : cercomère ; GC : granulations calcaires ; VA : ventouse apicale ; VL : ventouse latérale. 
granulations calcaires apparaissent dans le tégument. Au bout de 14 jours (nombre d'observations : 10 ; prévalence : 100 ; intensité : 4), les larves sont très mobiles et contractiles. Les granulations calcaires sont nombreuses et les cercomères ont complètement disparu. Les ventouses latérales et la ventouse apicale sont bien visibles (fig. 4). Les caractéristiques morphologiques moyennes de ces larves très contractiles sont présentées au tableau VI. L'examen de ces larves permet d'observer un appareil excréteur bien en place avec un réseau complexe de canaux.

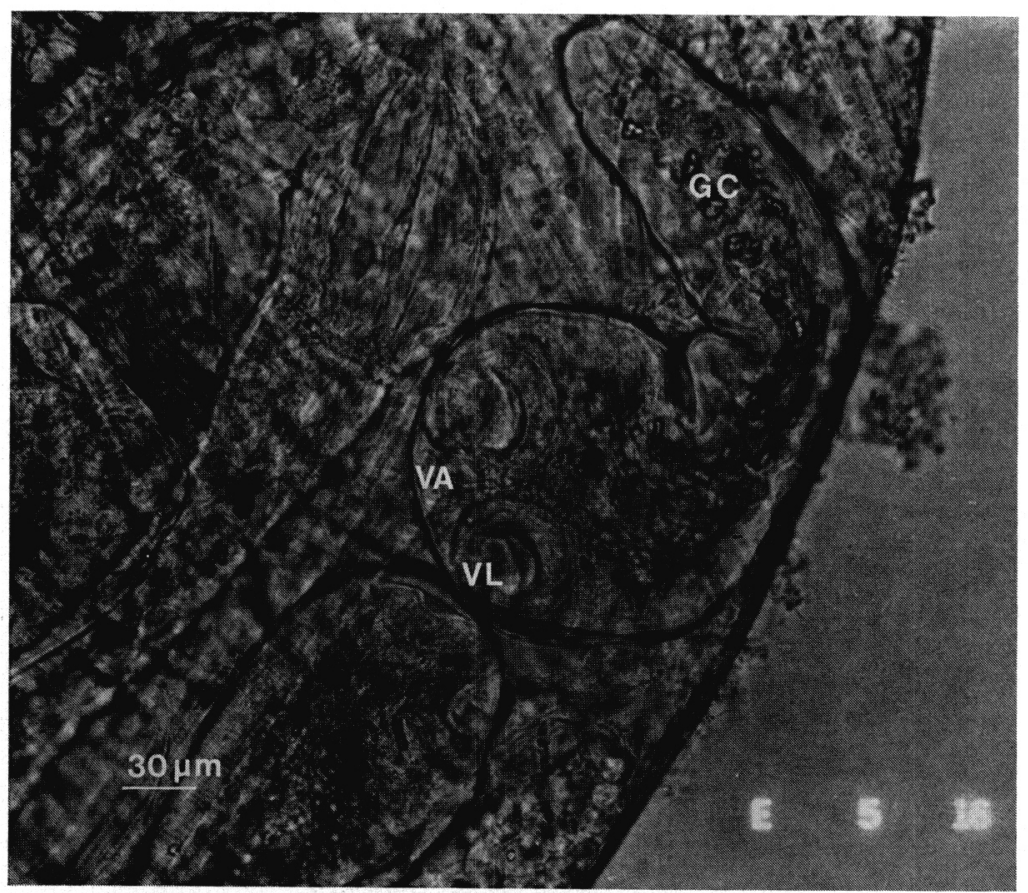

FIg. 4. - Larves de protéocéphale dans la cavité générale d'un Cyclops vicinus 14 jours après l'infestation. Légende identique à celle de la figure 3.

Tableau VI. - Caractéristiques morphologiques des larves observées chez Cyclops vicinus.

\begin{tabular}{lrc}
\hline & Ce travail & $\begin{array}{c}\text { Jarecka et Doby } \\
(1965)\end{array}$ \\
\hline Longueur & & $350 \mu \mathrm{m}$ \\
Largeur & $330-450 \mu \mathrm{m}$ & $100 \mu \mathrm{m}$ \\
Diamètre ventouses latérales & $100-120 \mu \mathrm{m}$ & $35-40 \mu \mathrm{m}$ \\
Diamètre ventouse apicale & $48-60 \mu \mathrm{m}$ & $30 \mu \mathrm{m}$ \\
\hline
\end{tabular}


3.3 - INFESTATION DE JEUNES CORÉgONES N'A YANT PAS ENCORE PASSÉ LEUR PREMIER ÉTÉ $\left(0^{+}\right)$

Un contrôle après quelques heures, de l'infestation de cinq poissons ne permit pas de mettre en évidence la présence de larves. Après 46 jours, l'autopsie des corégones n'a pas permis de déterminer une différence statistiquement significative entre l'infestation des corégones du lot infesté artificiellement (nombre d'observations : 20 ; prévalence : 5 ; intensité : 1 ) et celle du lot témoin (nombre d'observations : 30 ; prévalence : 0). Cependant, compte tenu du protocole utilisant des poissons nés en pisciculture, élevés uniquement sur granulés et dans une eau de source, on peut admettre que la prévalence observée dans le lot infesté artificiellement est probante. Les mensurations de la larve observée sont présentées au tableau VII.

Tableau VII. - Caractéristiques morphologiques des juvéniles observćes dans la zone intestinale des corégones $0^{+}, 46$ jours après l'infestation.

Longueur

Largeur du scolex

Diamètre ventouses latérales

Diamètre ventouse apicale
920 à $1600 \mu \mathrm{m}$

170 à $190 \mu \mathrm{m}$

80 à $\quad 90 \mu \mathrm{m}$

60 à $\quad 65 \mu \mathrm{m}$

\section{Discussion}

Le matériel que nous avons utilisé pour notre expérimentation est semblable à celui décrit par Doby et Jarecka (1964) et Jarecka et Doby (1965). Quelques variations dans la structure et le comportement de la larve peuvent toutefois être notées : l'onchosphère est apparue immobile lorsqu'elle se trouve dans la cavité générale du Cyclops et les larves de 14 jours ne nous montrent pas de crochets résiduels. D’autre part, Jarecka et Doby (1965) sitent Cyclops abyssorum abyssorum et C. streenuus strenuus comme hôtes intermédiaires alors qu'il apparaît que Cyclops vicinus Ulianine, 1875 est le seul hôte intermédiaire du parasite étudié dans le lac Léman. La présence de ces trois espèces dans ce lac (Balvay, 1984), la détermination spécifique particulièrement délicate pour le genre Cyclops ainsi que la systématique en constante évolution pour ce genre, permettent d'expliquer les divergences observées sur ce point.

Les prévalences des larves chez les Cyclops infestés artificiellement indiquent la réussite de ce type d'expérimentation. Comme la densité de Cyclops semble avoir un grand rôle dans le succès de l'infestation, il est possible aussi d'envisager un rôle des paramètres température et degré de maturité des œufs et des larves. Ainsi, des pontes provoquées en juin montrent des œufs immatures ne parvenant pas à l'état de turgescence et mourant rapidement. De la même manière, des pontes provoquées en période de maturité dans une eau de faible température $\left(\mathrm{T}=12^{\circ} \mathrm{C}\right)$ ne permettent pas l'infestation de Copépodes. 
La valeur peu élevée de la prévalence observée chez les corégones 0 + s'explique par la faible quantité de Cyclops infestés survivants ayant servi de repas. Bien que faible, ce taux d'infestation prouve que le cycle complet du protéocéphale des corégones du Léman est possible au laboratoire.

Dans le milieu naturel, le cycle de ce parasite montre une phase de maturité sexuelle des individus allant de juin à novembre avec des œufs infestants pendant seulement une partie de la maturité anatomique. Les parasites adultes disparaissent ensuite pendant l'hiver. Seules peuvent être observées des juvéniles dont la structure est comparable à celle de la larve parasite des Cyclopides. Cette période de purge hivernale pourrait être due à différents facteurs comme la baisse de la température de l'eau et l'approche de la période de reproduction des corégones qui entraîne des modifications d'ordre physico-chimique chez les poissons (Dabrowski et Champigneulle, 1987). Ce cycle est comparable à celui de Proteocephalus stizostethi chez Stizostedion vitreum vitreum (Connor, 1953) qui est annuel et comporte une purge parasitaire en fin d'été-début de l'automne, la période de ponte se situant au printemps.

La présence de seulement deux hôtes dans le cycle du parasite étudié est confirmée par le fait que les corégones du lac Léman sont strictement microphages (Ponton et Gerdeaux, 1988), que tous les stades évolutifs peuvent être observés dans une même année chez des poissons d'une même classe d'âge et que les larves observées chez les Cyclops sont morphologiquement très semblables aux juvéniles parasites des jeunes corégones $0^{+}$. Si Cyclops vicinus est le seul hôte intermédiaire, on ne discerne pas alors la présence d'un stade cysticercoïde chez un hôte d'attente supplémentaire, comme c'est le cas pour Proteocephalus pinguis (Hunter, 1929) et P. tumidocollus (Wagner, 1957).

L'observation d'une infestation naturelle de jeunes corégones $0^{+}$en juin 1984 et 1985 (Morandi et Ponton, 1988) indique une survie des larves ou des œufs du parasite durant l'hiver. Comme Jarecka et Doby (1965) ont montré que la prévalence diminue dans les élevages de Cyclopides quand ces derniers subissent une mue, la survie durant l'hiver des larves de protéocéphales pourrait se faire soit chez des Cyclops adultes, soit chez des copépodites en diapause dans les sédiments du lac. La première hypothèse suppose une durée de vie de plusieurs mois des Cyclops adultes, ce qui est incompatible avec les données de la littérature scientifique (Balvay, comm. pers.). La diapause estivale des copépodites de Cyclops vicinus et sa levée progressive en hiver (Einsle, 1967 ; George, 1973) permettraient ainsi la constitution d'un stock de Cyclopides infestés qui seraient ensuite relargués progressivement dans le milieu tout au long de l'hiver.

Cette période hivernale permettrait de définir un point zéro dans la dynamique d'infestation du fait de l'évacuation des parasites adultes. A cette saison, les Cyclopides sont très abondamment représentés et ils constituent la nourriture principale des corégones (Jacobsen, 1982 ; Ponton, 1986). Cette période serait donc particulièrement intéressante pour une approche quantitative du flux trophique entre le plancton et les corégones du lac Léman. 
Remerciements. - Ce travail a bénéficié de l'aide financière du C. N. R. S.-P. I. R. E. N. Maîtrise des ressources ichtyologiques.

\section{RÉFÉRENCES}

Balvay G. : Les Entomostracés du Léman. Schweiz. Z. Hydrol., 1984, 46, 230-246.

Buttiker B. : La faune piscicole in Le Léman, Synthèse des travaux de la CIPEL, 1957-1982, $1984,315-323$

Chaubeau-Duffour C., Morandi H. : Pathologie des poissons du lac Léman. Première approche. Rev. Med. Vet., 1984, 6, 367-378.

Connor R. S. : A study of the seasonal cycle of a Proteocephalan Cestode, Proteocephalus stizostheti Hunter et Bangham, found in the yellow Pikeperch, Stizostedion vitreum vitreum (Mitchill). J. Parasitol., 1953, 39, 621-624.

Doвy J. M., Jarecka L. : Redescription d'un Proteocephalus (Cestode) parasite du poisson Coregonus fera en provenance du lac Léman. Problèmes posés par la diagnose spécifique des Cestodes du genre Proteocephalus. Bull. Soc. Zool. France, 1964, 89, 675-687.

Dabrowski K. R., Champigneulle A. : Chemical composition of whitefish (Coregonidae) from lake Leman during spawning. Proc. V Congr. Europ. Ichthyol., Stockholm, 1985, 1987, $335-338$.

Einsle U. : Die ausseren Bedingungen der Diapause planktisch lebender Cyclops Arten. Arch. Hydrobiol., 1967, 63, 387-403.

Freze V. I. : Proteocephalata in Fish, Amphibians and Reptiles. Essentials of Cestodology, Vol. V. Israel Progr. Scient. Translations, Jerusalem, 1969, 1965, 597 p.

George D. G. : Diapause in Cyclops vicinus. Ö̈kos, 1973, 24, 137-142.

Hunter G. W. : Life history studies on Proteocephalus pinguis La Rue. Parasitology, 1929, 21, 487-496.

Iyeshio Y. P., Anikiyeva L. V., Bushman L. G. : The use of parasitological data for assessment of the food requirements of the european Cisco Coregonus albula. J. Ichthyol., 1981, 21, 172177.

JA CoBsen O. J. : A review of food and feeding habits in coregonid fishes. Pol. Arch. Hydrobiol., $1982,29,179-200$.

Jarecka L., Doby J. M. : Contribution à l'étude du cycle évolutif d'un Cestode du genre Proteocephalus parasite de Coregonus fera en provenance du lac Léman. Ann. Parasitol. Hum. Comp., 1965, 40, 433-443.

Margolis L. G. W., Esch J. C., Holmes A. M., Kuris, Schad G. A. : The use of ecological terms in parasitology (Report of and ad hoc commitee of the American Society of Parasitologists). J. Parasitol., 1982, 68, 131-133.

Ponton D. : Croissance et alimentation de deux poissons planctonophages du lac Léman : le corégone (Coregonus schinzii palea) et le gardon (Rutilus rutilus). Thèse $3^{\mathrm{e}}$ Cycle, Univ. Lyon I, $1986,158 \mathrm{p}$.

Ponton D., Gerdeaux D. : Quelques aspects de l'alimentation de deux poissons planctonophages du lac Léman : le corégone (Coregonus schinzii palea Cuv. et Val.) et le gardon (Rutilus rutilus (L.)). Bull. Fr. Pêche Pisc., 1988, 308, 11-23.

Morandi H., Pcnton D. : Contribution à l'étude d'un Cestode Proteocephalidae parasite du corégone du lac Léman. Évaluation de son rôle de marqueur biologique de la chaîne trophique. Rapport partiel C. N. R. S./P. I. R. E. N. Maîtrise des ressources ichtyologiques, $1988,20 \mathrm{p}$.

Wagner E. D. : The life history of Proteocephalus tumidocollus Wagner, 1953 (Cestoda) in Rainbow Trout. J. Parasitol., 1954, 40, 489-498. 